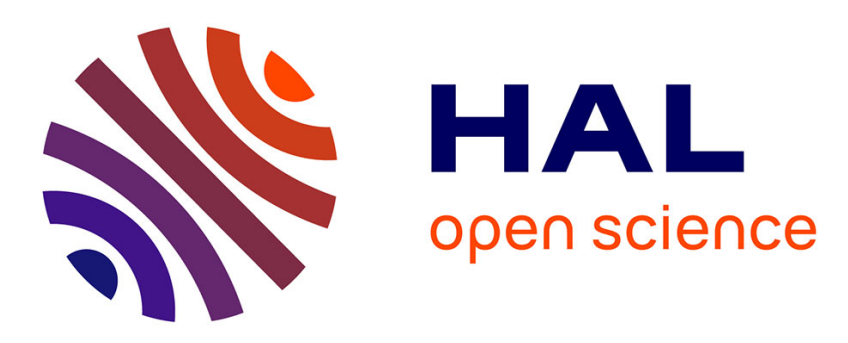

\title{
Methodology for extraction of space charge density profiles at nanoscale from Kelvin probe force microscopy measurements
}

Christina Villeneuve-Faure, Laurent Boudou, Kremena Makasheva, G. Teyssedre

\section{To cite this version:}

Christina Villeneuve-Faure, Laurent Boudou, Kremena Makasheva, G. Teyssedre. Methodology for extraction of space charge density profiles at nanoscale from Kelvin probe force microscopy measurements. Nanotechnology, 2017, 28 (50), pp.505701. 10.1088/1361-6528/aa9839 . hal-02324308

\section{HAL Id: hal-02324308 \\ https://hal.science/hal-02324308}

Submitted on 1 Nov 2019

HAL is a multi-disciplinary open access archive for the deposit and dissemination of scientific research documents, whether they are published or not. The documents may come from teaching and research institutions in France or abroad, or from public or private research centers.
L'archive ouverte pluridisciplinaire HAL, est destinée au dépôt et à la diffusion de documents scientifiques de niveau recherche, publiés ou non, émanant des établissements d'enseignement et de recherche français ou étrangers, des laboratoires publics ou privés. 


\title{
Methodology for extraction of space charge density profiles at nanoscale from Kelvin probe force microscopy measurements
}

\author{
C Villeneuve-Faure ${ }^{-}$, L Boudou, K Makasheva ${ }^{\circledR}$ and G Teyssedre \\ LAPLACE (Laboratoire Plasma et Conversion d'Energie), Université de Toulouse, CNRS, INPT, UPS, Bat \\ 3R3, 118 route de Narbonne, F-31062 Toulouse Cedex 9, France \\ E-mail: christina.villeneuve@laplace.univ-tlse.fr
}

Received 27 July 2017, revised 24 October 2017

Accepted for publication 3 November 2017

Published 21 November 2017

\begin{abstract}
To understand the physical phenomena occurring at metal/dielectric interfaces, determination of the charge density profile at nanoscale is crucial. To deal with this issue, charges were injected applying a DC voltage on lateral Al-electrodes embedded in a $\mathrm{SiN}_{x}$ thin dielectric layer. The surface potential induced by the injected charges was probed by Kelvin probe force microscopy (KPFM). It was found that the KPFM frequency mode is a better adapted method to probe accurately the charge profile. To extract the charge density profile from the surface potential two numerical approaches based on the solution to Poisson's equation for electrostatics were investigated: the second derivative model method, already reported in the literature, and a new 2D method based on the finite element method (FEM). Results highlight that the FEM is more robust to noise or artifacts in the case of a non-flat initial surface potential. Moreover, according to theoretical study the FEM appears to be a good candidate for determining charge density in dielectric films with thicknesses in the range from $10 \mathrm{~nm}$ to $10 \mu \mathrm{m}$. By applying this method, the charge density profile was determined at nanoscale, highlighting that the charge cloud remains close to the interface.
\end{abstract}

Keywords: KPFM, dielectric thin film, space charge measurement

(Some figures may appear in colour only in the online journal)

\section{Introduction}

Space charge injection and transport in dielectric materials are the main causes of failure in many electrical systems. Such issues can arise at different scales in radio-frequency microelectromechanical systems with electrostatic actuation [1], CMOS with a thin gate dielectric film [2] or insulation for HVDC systems [3, 4]. Failure in the insulating material generally leads to a loss of control in the system, requiring a premature replacement. Consequently, the understanding of charge build-up and transport in dielectric materials is crucial for improving their performance and reliability. Classical space charge probing methods [5] as, for example, the pulse electroacoustic [6] or light intensity modulation methods [7] have demonstrated in-depth resolution of, at best, a few microns. These techniques are well adapted to probe thick dielectric films (more than $20 \mu \mathrm{m}$ ), but fail in the attempt to investigate charges in thin dielectric films (less than a few microns thickness) [8]. For twenty years now, derivative methods from atomic force microscopy (AFM) have been increasingly used to probe charges in thin dielectric films [9-11]. Indeed, the nanoscale lateral resolution offered by AFM is promising to overcome the limits of in-depth resolution of classical space charge probing methods.

Among the existing electrical modes derivative to AFM, Kelvin probe force microscopy (KPFM) and electrostatic force microscopy (EFM) have been extensively used to determine the charge injection and transport in thin dielectric films [9-11]. Charges are injected in the dielectric film under bias voltage by using an AFM tip [12-14] or through lateral electrodes [15-19]. Concerning charge injection by lateral electrodes, for the time being only a few studies are available 


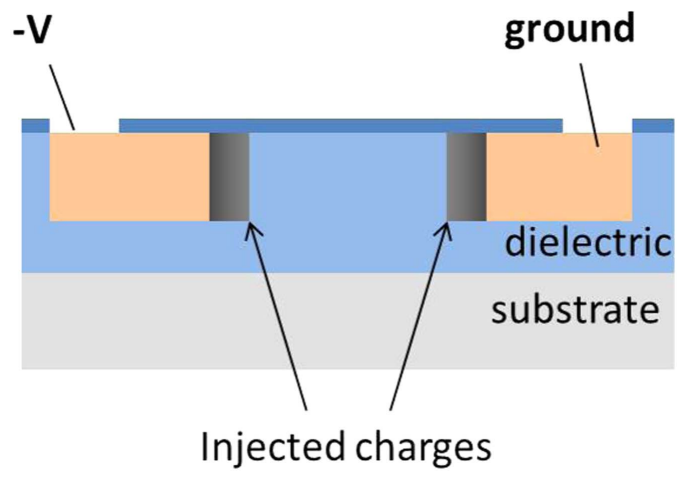

Figure 1. Sample structure-cross-view.

in the literature. They mainly address the charge injection phenomenon in a qualitative way without determining charge density profiles. Very recently, a method based on a numerical procedure for treatment of the recorded signal in relation to the solution of Poisson's equation was proposed to extract space charge profiles between two lateral electrodes [20, 21]. However, it does not offer a clear demonstration of the validity of the approach for thin dielectric films.

The objective in this paper is to propose a combined experimental and modeling method adapted to determine the space charge density profile at nanoscale in a thin dielectric layer through KPFM measurement by using lateral electrodes embedded in the layer. To that end two modeling methods were investigated. The first one is based on the second derivative of the surface potential profile using Poisson's equation, as described in Mortreuil et al [19]. The second one relies on surface potential computing using the finite element model (FEM). This paper is divided into three parts. The first part is dedicated to the experimental procedure. In the second part the two modeling approaches are described and the methodology to extract space charge density profiles from the experimental results is presented. In the last part, each technique is compared in terms of performance and sensitivity to sample geometry and material characteristics.

\section{Experiments}

The dielectric films used in this study were $300 \mathrm{~nm}$ thick $\mathrm{SiN}_{x}$ layers. They were deposited by high frequency plasma enhanced chemical vapor deposition [22] on low resistivity silicon wafers. Embedded aluminum electrodes were processed by a lift-off process using a $2.5 \mu \mathrm{m}$ thick NLOF photoresist. This resin was deposited and patterned by photolithography. Then, the $\mathrm{SiN}_{x}$ was chemically etched to a depth of $70 \mathrm{~nm}$ and filled in by aluminum. Thanks to this twostep process a close metal/dielectric contact is assured. Finally, a $5 \mathrm{~nm} \mathrm{SiN}_{x}$ passivation layer was deposited over the embedded electrodes to avoid discharges during polarization. This process provides a step height between the electrode and the dielectric layer less than $5 \mathrm{~nm}$, which prevents topography artifacts during KPFM measurement. Figure 1 represents a schematic cross-view of the final structure. Structures with

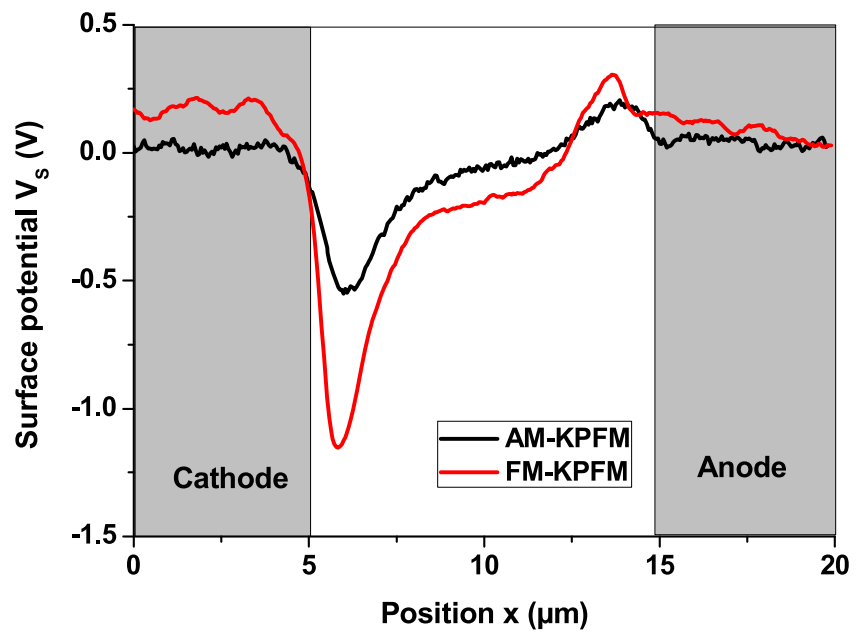

Figure 2. Comparison of surface potential profiles measured by the AM-KPFM and FM-KPFM techniques for an inter-electrode distance of $10 \mu \mathrm{m}$ after $1 \mathrm{~h}$ of polarization ( $-40 \mathrm{~V}$ applied to the left electrode and ground applied to the right one during polarization).

inter-electrode distances ranging from $5 \mu \mathrm{m}$ to $40 \mu \mathrm{m}$ were designed and elaborated.

The electric field was induced by applying bias on the electrodes for one hour (figure 1): ground on one electrode and $-V$ on the other one. The silicon substrate backside was set to the ground during the experiment. The surface potential was measured immediately after the polarization step. The surface potential was measured with a Bruker Multimode 8 set-up using Pt-coated silicon tip. Measurements were performed using KPFM in amplitude (AM-KPFM) and frequency (FM-KPFM) modes. For AM-KPFM a $10 \mathrm{~nm}$ lift height was used whereas FM-KPFM was performed in a single-pass mode (topography and surface potential were measured during the same scan). All measurements were done under a dry $\mathrm{N}_{2}$ atmosphere after sample conditioning for $4 \mathrm{~min}$ at $120^{\circ} \mathrm{C}$ to remove the water layer adsorbed on the surface. The measurement step was fixed to $40 \mathrm{~nm}$.

$\mathrm{AM}$ and FM measurements were not performed at the same time or for the same polarization step due to the necessary calibration step, which prevents the switching between measurement technique in the course of measurement. However, results reproducibility was checked to ensure that the AM and the FM potential profiles were comparable.

\section{Methodology to extract the charge density profile}

\subsection{KPFM measurements}

Figure 2 compares the surface potential profiles obtained by AM-KPFM and FM-KPFM after polarization. During these measurements both electrodes were at floating potential. For both measurement methods, two peaks appeared close to the metal/dielectric interfaces; a negative peak close to the cathode and a positive peak close to the anode side. The maximum potential $V_{\mathrm{m}}$ and the full-width at half maximum (FWHM) were extracted for each peak. The obtained values 
(a)

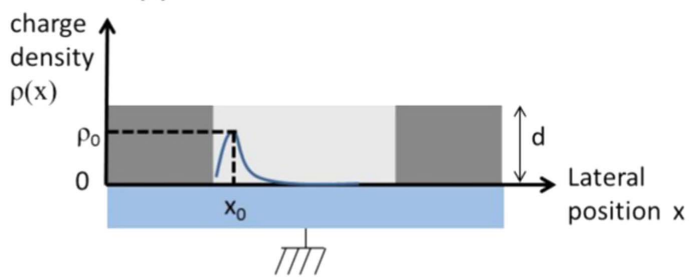

$\mathrm{SiN}_{\mathrm{x}}$ with charge density $\rho(\mathrm{x})$

$\mathrm{SiN}_{\mathrm{x}}$ without charge

Floating electrodes (b)

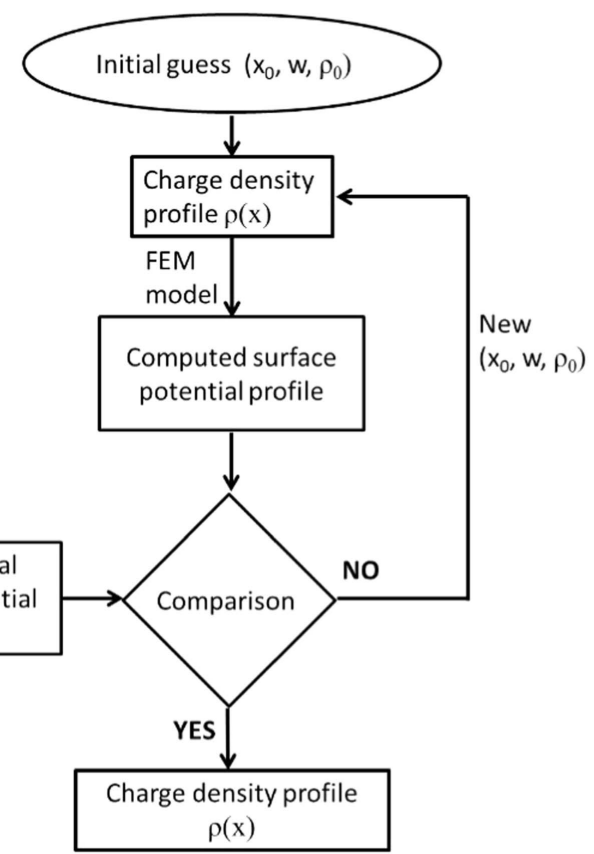

Figure 3. (a) Schematic representation of the modeled structure and (b) FEM based model flow chart.

are reported in table 1 . When using FM-KPFM the determined potential peak intensity was higher and the FWHM was lower in comparison with the results obtained by AMKPFM. Moreover, the measured signal to noise ratio was improved. This is due to the fact that the FM-KPFM sensitivity and spatial resolution are higher than those for AMKPFM [23]. Consequently, one can state that the FM-KPFM is better adapted to probe charges at local scale because the measured surface potential is closer to the real one, in accordance with the results reported and discussed by Cohen et al [24]. In the following the surface potential was measured by FM-KPFM.

\subsection{Modeling approaches}

To extract the space charge density profile from the surface potential profile measured by FM-KPFM two modeling approaches were explored. They are both based on the resolution of Poisson's equation for electrostatics. The first approach to extract the charge density profile $\rho(x)$, already presented by Mortreuil et al [19,25], is a 1D model giving directly $\rho(x)$ as a second derivative of the measured surface potential:

$$
\rho(x)=-\varepsilon_{0} \varepsilon_{\mathrm{r}} \frac{\mathrm{d}^{2} V_{\mathrm{S}}}{\mathrm{d} x^{2}},
$$

where $\varepsilon_{0}$ is the vacuum permittivity, $\varepsilon_{\mathrm{r}}$ is the relative permittivity of the dielectric material, $V_{\mathrm{S}}(x)$ is the measured surface potential, and $x$ is the lateral position. We call this approach the second derivative model (SDM). Applying equation (1) to the surface potential implicitly supposes that the measured potential is equivalent to that along the thickness direction in 1D with infinite planar geometry for the electrodes and the dielectric. Consequently, the SDM does not take into account air/dielectric and substrate/dielectric interface influence.

The best derivation is obtained using a step of $\mathrm{d} x=80 \mathrm{~nm}$, which is actually twice the measurement step. Refining the differentiation step is needed when treating the FM-KPFM signal as the latter is steeper. This kind of processing becomes possible as the recorded signal is less noisy for FM-KPFM compared to AM-KPFM. The approach described here differs from the one reported by Faliya et al [21] by the fact that no preliminary signal treatment (smoothing, fitting or filtering) was applied to the KPFM experimental raw data. In our configuration the recorded data are with high signal to noise ratio and do not require any additional signal processing.

The second method is based on the use of the FEM of COMSOL Multiphysics ${ }^{\circledR}$ software. The resulting 2D model represents the sample structure as shown in figure 3(a), surrounded by an air box of dimensions large enough to avoid edge effects. The relative dielectric permittivity of $\mathrm{SiN}_{x}$ is taken as 7.5, which is given for a frequency of $1 \mathrm{kHz}$ at $23^{\circ} \mathrm{C}$. The silicon substrate backside is set to the ground. The electrodes remain at floating potential, as in the experiment. The flow chart of the FEM approach is shown in figure 3(b). The model is initialized with generation of charge density clouds having Gaussian distribution of the charge density at both electrodes (figure 3(a)). For positive and negative charges, the density is expressed as:

$$
\rho(x)=\rho_{0} \exp \left(-\frac{\left(x-x_{0}\right)^{2}}{0.36 W^{2}}\right)
$$

where $\rho_{0}$ is the maximum value (positive for holes and negative for electrons), $x_{0}$ is the maximum position and $W$ is the FWHM of the charge cloud. Poisson's equation is solved in two dimensions in the dielectric layer and in the surrounding air to determine the potential distribution $V(x, z)$ in 

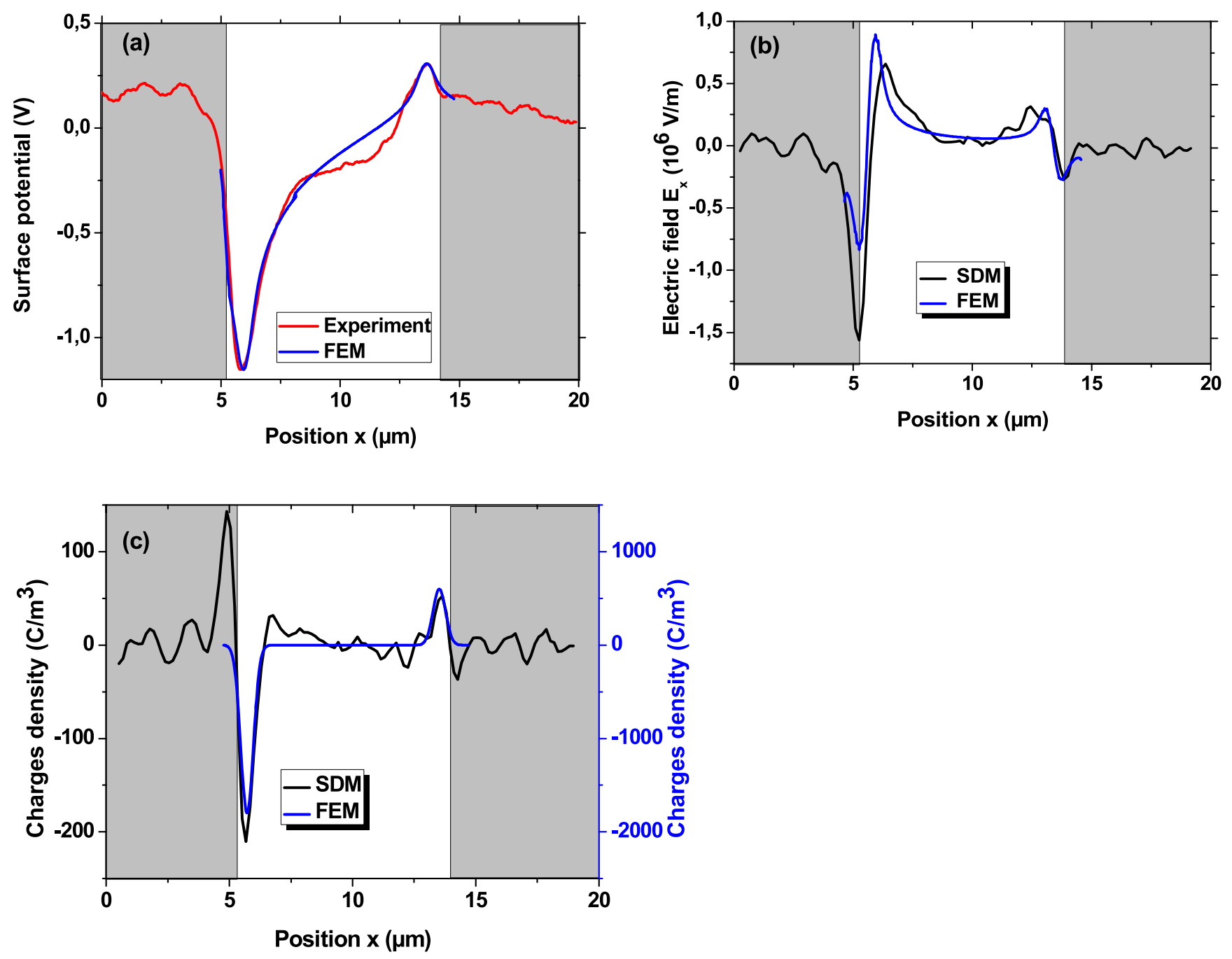

Figure 4. Comparison of (a) surface potential, (b) electric field distribution in the $x$-direction, $E_{x}$ and (c) charge density profiles extracted from the surface potential profile measured by FM-KPFM using the SDM and the FEM models.

the structure :

$$
\frac{\mathrm{d}^{2} V}{\mathrm{~d} x^{2}}+\frac{\mathrm{d}^{2} V}{\mathrm{~d} z^{2}}=-\frac{\rho(x, z)}{\varepsilon_{0} \varepsilon_{\mathrm{r}}(x, z)} .
$$

This approach permits us to take into account the real geometrical constraints and the influence of the dielectric layer interfaces at the substrate and the surrounding air. The initial values for $x_{0}$ and $W$ correspond to the experimental values of the surface potential maximum position and the FWHM respectively. $\rho_{0}$ is initiated at $1000 \mathrm{C} \mathrm{m}^{-3}$. The computed surface potential profile is then compared to the experimentally obtained profile of the surface potential (figure 3(b)). This comparison is done using three criteria: (i) one on the maximum potential, $\Delta V_{\mathrm{m}}$, (ii) one on the FWHM, $\Delta_{\text {FWHM }}$, and (iii) one on the position $\Delta x_{0}$, which correspond to the differences between the experimental and computed values of maximum intensity, FWHM and position of the peak of the potential profile, respectively. As criteria to stop the iterations, we accepted a difference between computed and experimental potential profiles of less than $8 \%$ on the maximum intensity and less than $4 \%$ on the FWHM and on
Table 1. Maximum intensity and FWHM of negative and positive surface potential peaks determined from AM-KPFM and FM-KPFM measurements.

\begin{tabular}{lccccc}
\hline & $\begin{array}{c}\text { Negative peak (close to } \\
\text { the cathode) }\end{array}$ & & \multicolumn{2}{c}{$\begin{array}{c}\text { Positive peak (close to } \\
\text { the anode) }\end{array}$} \\
\cline { 2 - 3 } \cline { 5 - 6 } & $\begin{array}{c}\text { Maximum } \\
V_{\mathrm{m}}(\mathrm{V})\end{array}$ & $\begin{array}{c}\text { FWHM } \\
(\mu \mathrm{m})\end{array}$ & & $\begin{array}{c}\text { Maximum } \\
V_{\mathrm{m}}(\mathrm{V})\end{array}$ & $\begin{array}{c}\text { FWHM } \\
(\mu \mathrm{m})\end{array}$ \\
\hline AM-KPFM & -0.65 & 1.9 & & 0.18 & 1.6 \\
FM-KPFM & -1.18 & 1.8 & & 0.32 & 1.4 \\
\hline
\end{tabular}

the position. The selected criteria correspond to the resolution of the KPFM technique [26].

In case of discrepancy, $\rho_{0}, x_{0}$ and $\mathrm{W}$ for the positive and negative charges distributions are adjusted. If the difference is less than $10 \%$, the parameter under iteration is modified by $5 \%$, otherwise it is modified by $10 \%$. The iteration procedure is maintained until complete matching between the computed and the measured surface potential profile is achieved (figure 3(b)).

Less than ten iterations are typically sufficient to fit. The charge density profile is then extracted from the calculated 


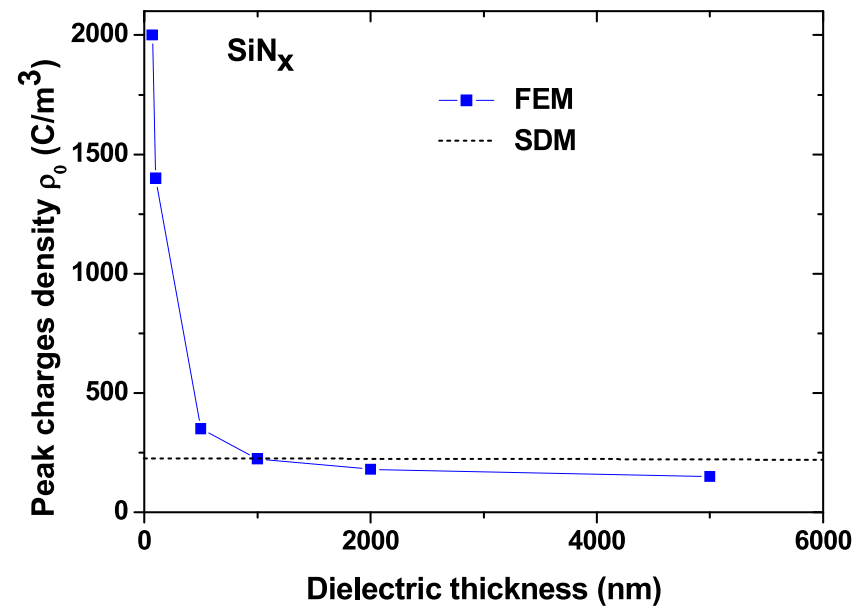

Figure 5. Evolution of the maximum charge density as function of dielectric layer thickness obtained for $\mathrm{SiN}_{x}$ films after using a surface potential profile with maximum of $1.18 \mathrm{~V}$ and FWHM of $1.8 \mu \mathrm{m}$.

surface potential profile. The main advantages of this approach are that it takes into account the limited thickness of the dielectric layer $(d)$ and offers identification of the real extension of the charges cloud in the volume.

\section{Results and discussion}

\subsection{Comparison of the modeling approaches}

The applicability of the two modeling approaches is demonstrated using the experimental FM-KPFM surface potential profile shown in figure 2 . The experimental conditions are as follows: $10 \mu \mathrm{m}$ inter-electrode distance, polarization time of $1 \mathrm{~h}$ with $-40 \mathrm{~V}$ applied to the left electrode and ground applied to the right one.

Figure 4(a) represents the surface potential profile as recorded by FM-KPFM on $\mathrm{SiN}_{x}$ thin film and the one obtained after FEM. Figure 4(b) compares electric field profiles extracted from the recorded surface potential profiles (figure 2) using the two numerical methods, as explained in the previous section. The results emphasize that the surface potential computed by the FEM adjusted accurately the experimental profile, except for a region far from the electrodes (figure 4(a)). The electric field profile is nearly the same for the two methods (figure 4(b)). Figure 4(c) represents a comparison of the charge density profiles extracted with each method. As for the electric field, both approaches provide charge density profiles with the same shape. For both models, the charge clouds are narrow and remain located close to the electrodes (maximum is located at $600 \mathrm{~nm}$ in the dielectric). The charge clouds exhibit the same shape for both models. This implies that they have nearly the same influence on the spatial resolution. The observed oscillations on the charge profile determined by the SDM are due to the noise on the surface potential profile, which is emphasized by the two derivative steps method. Moreover, the main feature is the fact that the FEM method provides charge density magnitude increased by a factor of ten compared to the SDM method.
The main difference between the two models is that the SDM model considers an infinite structure whereas the FEM model reproduces the real 2D-distribution considering the sample geometry. Indeed, the SDM allows extraction of only the electric field profile in the $x$-direction whereas the FEM computes the electric field intensities in the $x$ - and in the $z$ directions, i.e. $E_{x}$ and $E_{z}$. The contribution in the $z$-direction comes from the finite dimension of the dielectric film. Indeed, FEM results highlight that $E_{z}$ is non-null close to the charge clouds and reaches a maximum of around $1 \times 10^{5} \mathrm{~V} \mathrm{~m}^{-1}$ close to the substrate.

The FEM model was applied to investigate the influence of the dielectric film thickness on the charge density determination. For different dielectric layer thicknesses $d$ (figure 3(a)), the charge density was determined to obtain a potential profile for negative charges close to the one shown on figure 2 (potential maximum at $1.18 \mathrm{~V}$ and $\mathrm{FWHM}=$ $1.8 \mu \mathrm{m}$ ). Figure 5 represents the maximum charge density $\rho_{0}$ as a function of the dielectric layer thickness for $\mathrm{SiN}_{x}$ films with different thicknesses. The results emphasize that the determined maximum charge density is strongly influenced by the dielectric thickness. It decreases with increasing the thickness. It shows that a higher charge amount is necessary to provide a given voltage potential when the film is thinner. In addition, for film thicknesses over $1 \mu \mathrm{m}$, a mild evolution in the charge density is observed. The FEM model provides nearly the same amount of charge as the SDM model for large thicknesses. This is probably due to the fact that the $E_{z}$ contribution decreases with the dielectric thickness increase because of the $E_{z}$ maximum close to the substrate.

These results highlight that the SDM model provides accurate results in terms of charge cloud shape but underestimates the charge density in the case of thin films (less than $1 \mu \mathrm{m})$. Consequently, the FEM 2D model should be preferred to the SDM 1D model when probing thin films. A drawback of the FEM model reported here is that a priori knowledge in the shape of the charge clouds is necessary, in the current stage of development, whereas the SDM does not necessitate such information. A combination of the two methods can be a way to solve the problem. Indeed, the SDM model can be used to find the initial values of $x_{0}$ and $W$ to reduce the number of iterations on these parameters. It can also be used to provide a guess on the shape of the charge cloud. In addition, the SDM allows a charge peak on the electrodes to appear, which can be interpreted as resulting from the influence of charges generated on the electrodes. This could also be considered in a future hybrid FEM-SDM model.

\subsection{Influence of the initial surface potential profile}

In the processing of samples, the deposition of a $5 \mathrm{~nm}$ passivation layer for the structure was systematically adopted. Without a passivation layer over the electrodes, the measured initial surface potential is not flat, as shown in figure 6(a). This non-flat profile stems from the difference between the $\mathrm{Al}$ work function and the $\mathrm{SiN}_{x}$ surface potential. After polarization, the surface potential is modified only close to the cathode (i.e. the surface potential is now a superposition of 

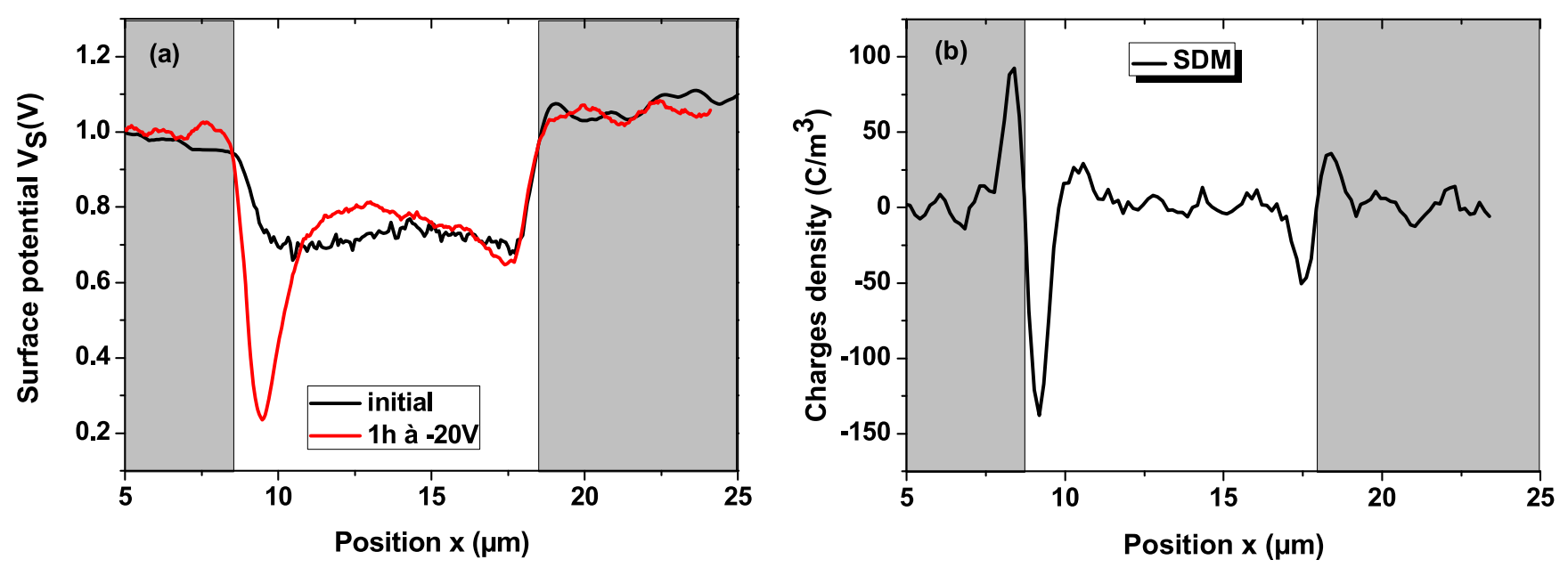

Figure 6. (a) Evolution with time of the surface potential profile measured by FM-KPFM before and after $1 \mathrm{~h}$ polarization $(-20 \mathrm{~V}$ applied to the left electrode and ground applied to the right one). In this case the Al-electrodes were not passivated. (b) Corresponding charge density profiles extracted using the SDM method.

the initial profile and the potential induced by injected charges). To extract the charge density two ways are available to proceed: (1) to correct the obtained profile by subtraction of the initial profile in order to avoid artifacts due to the initial non-flat profile or (2) to use directly the rough surface potential profile after polarization. Appling the SDM method to a corrected profile provides non-exploitable results because of the high noise level. When the SDM method is applied to the potential profile recorded on a rough surface it provides a charge density profile as the one depicted in figure 6(b). The charge density profile exhibits negative charges close to both electrodes. However, the electrons are injected close to the cathode due to applied negative bias. For the anode side, positive charges should be injected whereas the SDM method shows electron injection. This electron injection is only an artifact due to the shape of the initial surface potential (i.e. the surface potential difference between $\mathrm{SiN}_{x}$ and $\mathrm{Al}$ ). Indeed, the surface potential profile is not really modified close to the anode after polarization. Consequently, the SDM method is not adapted for use when the initial surface potential is not flat.

This drawback is avoided when using the FEM approach. Indeed, as the adjusting loop works with many parameters (position, potential maximum and FWHM), the FEM is less sensitive to noise and can be applied successfully on the corrected profile.

\subsection{Influence of the material composition through the dielectric permittivity: theoretical considerations}

In the previous section we demonstrated that for $\mathrm{SiN}_{x}$ dielectrics (relative permittivity $\varepsilon_{\mathrm{r}}=7.5$ ) the FEM and SDM models provide similar results for layer thicknesses larger than $1 \mu \mathrm{m}$. To investigate the influence of material composition through the dielectric permittivity, a theoretical surface potential profile with $1 \mathrm{~V}$ of maximum potential located at $2 \mu \mathrm{m}$ from the electrode with a FWHM of $1.8 \mu \mathrm{m}$ was considered. We considered thin layers with different dielectric permittivities $\left(\mathrm{SiO}_{2} \varepsilon_{\mathrm{r}}=3.9, \mathrm{Al}_{2} \mathrm{O}_{3} \varepsilon_{\mathrm{r}}=9, \mathrm{La}_{2} \mathrm{O}_{3} \varepsilon_{\mathrm{r}}=30\right.$,
$\mathrm{TiO}_{2} \varepsilon_{\mathrm{r}}=80$ ) [27]. Using this theoretical profile, both models were used to compute the corresponding charge profiles as a function of the dielectric film thickness. Figure 7(a) represents the maximum charge density $\rho_{0}$ as a function of the dielectric layer thickness for $\mathrm{TiO}_{2}$ film (relative permittivity $\varepsilon_{\mathrm{r}}=80$ ). As previously, the results emphasize that the determined maximum density is strongly influenced by the dielectric thickness when applying the FEM model. Moreover, the FEM and SDM models provide the same results only for thicknesses around $250 \mathrm{~nm}$. For other thicknesses, the difference is very strong. It might be doubled in the saturation region or even tripled when it goes for very thin films. This can be explained by the fact that when the dielectric permittivity is high, the electric field is less confined in the dielectric layer and the interfaces have stronger influence on the $E_{z}$ contribution for thin layers. Figure 7(b) summarizes the absolute values of the charge density difference between the two models normalized by the charge density extracted from the SDM. The quantity is plotted as a function of the film thickness for materials with different dielectric permittivities. For simplicity we introduce charge density deviation (CDD), which reads:

$$
\mathrm{CDD}(\%)=\frac{\operatorname{abs}\left(\rho_{0, \mathrm{SDM}}-\rho_{0, \mathrm{FEM}}\right)}{\rho_{0, \mathrm{SDM}}} \times 100 .
$$

$\mathrm{CDD}=0$ corresponds to the dielectric thickness for which both models provide the same maximum amount of charge. For dielectrics with low relative permittivity (less than 7.5, corresponding to the experimentally investigated $\mathrm{SiN}_{x}$ in this work) and thicknesses larger than $1 \mu \mathrm{m}$ the two models provide a charge amount in the CDD limit of 50\%. By contrast, if the dielectric thickness is less than $1 \mu \mathrm{m}$, the CDD increases very rapidly and can reach discrepancies between the two models of more than $500 \%$. For middle- and high- $k$ materials (relative dielectric permittivity higher than 7.5) the CDD is important for almost all film thicknesses, although it does not reach values as high as for thin dielectric layers with lower permittivity. With respect to the dielectric relative 

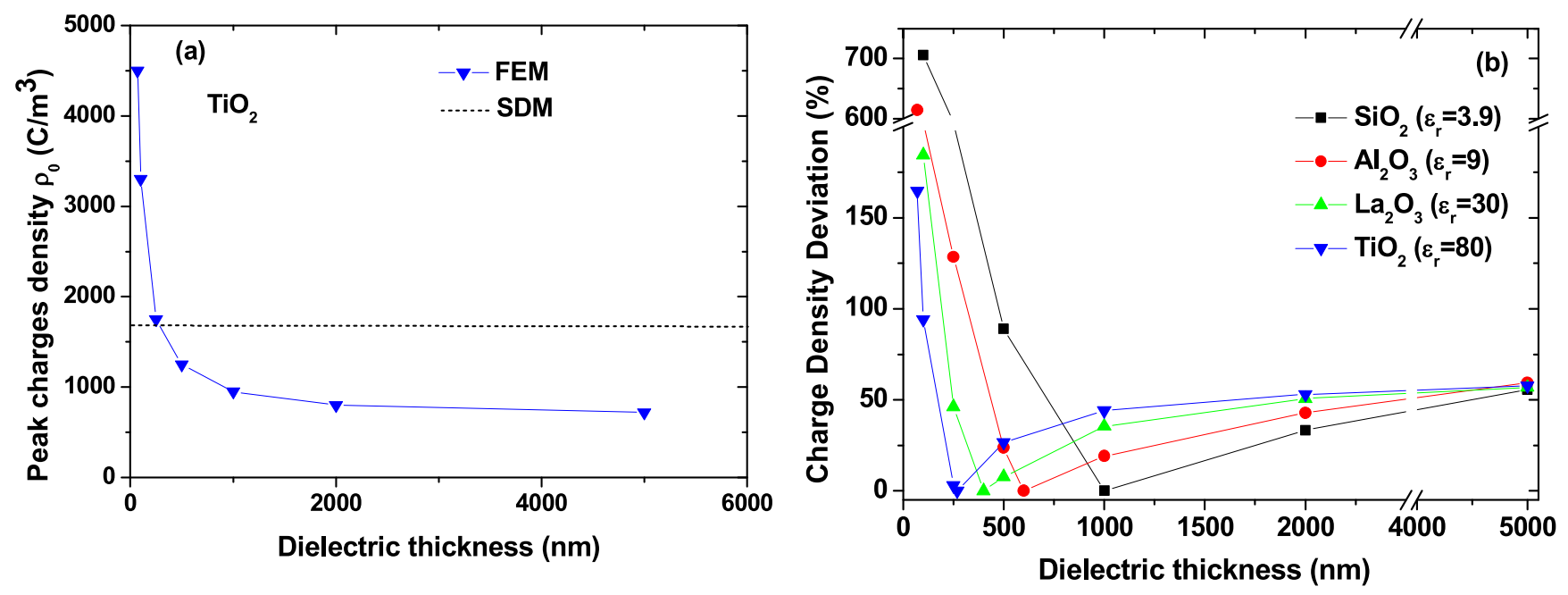

Figure 7. (a) Evolution of charge density as function of dielectric layer thickness obtained with a surface potential profile with a maximum of $1 \mathrm{~V}$ and FWHM of $1.8 \mu \mathrm{m}$ for a $\mathrm{TiO}_{2}$ layer (relative permittivity $\varepsilon_{\mathrm{r}}=80$ ). (b) Evolution of the $\mathrm{CDD}$ as a function of the dielectric film thickness for different dielectrics.

permittivity, increasing the permittivity of the dielectric layer fixes the zero CDD at lower thickness. This suggests that the span of application of the SDM is increased towards thin layers although the CDD remains at the level of around 50\%, which should correspond to a semi-infinite medium for FEM. However, the above analysis confirms that the FEM model is more appropriate for extracting charge profiles from the surface potential measurement.

\section{Conclusion}

In this paper, a methodology to extract charge density profile from surface potential profile was developed and validated. It is based on surface potential measurements by FM-KPFM followed by FEM modeling considering the real sample geometry. According to the obtained results the FEM method appears more robust than the SDM approach proposed in previous studies. The FEM method is $2 \mathrm{D}$ and offers the possibility of determining the space charge profile at nanometer scale after electrode polarization. It was found that for $\mathrm{SiN}_{x}$ dielectric layers the charges cloud is narrow $(\mathrm{FWHM}=1.8 \mu \mathrm{m})$ and remains located close to the electrodes (at around $600 \mathrm{~nm}$ ). Application of the FEM method appears more robust to noise and artifacts, and it also considers the real geometry of the sample. In addition, it is more sensitive with respect to the dielectric layers with different relative permittivity than the SDM approach. A drawback of the method is that in its present version it requires a priori knowledge of the shape of the charge distribution. The FEM method presented here will be further used to study charge injection and transport at metal/dielectric interfaces.

\section{Acknowledgments}

This work was partly supported by the French RENATECH network.

\section{ORCID iDs}

C Villeneuve-Faure 1 https://orcid.org/0000-0002-7959-2912

K Makasheva $\odot$ https://orcid.org/0000-0001-6113-3593

\section{References}

[1] Witvrouw A, Tilmans H A C and De Wolf I 2004 Materials issues in the processing, the operation and the reliability of MEMS Microelectron. Eng. 76245

[2] Kerber A, Cartier E, Degraeve R, Roussel P J, Pantisano L, Kauerauf T, Groeseneken G, Maes H E and Schwalke U 2003 Charge trapping and dielectric reliability of $\mathrm{SiO}_{2}-\mathrm{Al}_{2} \mathrm{O}_{3}$ Gate stacks with TiN electrodes IEEE Trans. Electron Dev. 501261

[3] Zhang Y, Lewiner J, Alquié C and Hampton N 1996 Evidence of strong correlation between space-charge buildup and breakdown in cable insulation IEEE Trans. Dielectr. Electr. Insul. 3778

[4] Milliere L, Makasheva K, Laurent C, Despax B, Boudou L and Teyssedre G 2016 Silver nanoparticles as a key feature of a plasma polymer composite layer in mitigation of charge injection into polyethylene under de stress J. Phys. D: Appl. Phys. 49015304

[5] Ahmed N H and Srinivas N N 1997 Review of space charge measurements in dielectrics IEEE Trans. Dielectr. Electr. Insul. 4644

[6] Maeno T and Fukunaga K 1996 High-resolution PEA charge distribution measurement system IEEE Trans. Dielec. Electr. Insul. 3754

[7] Petre A, Pham C D, Marty-Dessus D and Berquez L 2009 Three-dimensional space charge cartographies by FLIMM in electron irradiated polymers J. Electrostat. 67430

[8] Teyssedre G, Villeneuve C, Pons P, Boudou L, Makasheva K and Despax B 2012 Challenges in probing space charge at submicrometer scale Annual Report IEEE Conf. on Electrical Insulation and Dielectric Phenomena (CEIDP) p 234

[9] Ishii M 2010 Static states and dynamic behaviour of charges : observation and control by scanning probe microscopy J. Phys.: Cond. Matter. 22173001 
[10] Rezende C A, Gouveia R F, da Silva M A and Galembeck F 2009 Detection of charge distributions in insulator surfaces J. Phys. Condens. Matter 21263002

[11] Stevens G C and Baird P J 2005 Nano- and Meso-measurement methods in the study of dielectrics IEEE Trans. Dielec. Electr. Insul. 12979

[12] Tzeng S D and Gwo S 2006 Charge trapping properties at silicon nitride/silicon oxide interface studied by variabletemperature electrostatic force microscopy J. Appl. Phys. 100023711

[13] Sridhara K, Borowik L, Mariolle D, Chevalier N and Colonna J P 2012 Environment and baking influence on charge retention on silicon nitride charge trap layers J. Appl. Phys. 111023710

[14] Villeneuve-Faure C, Makasheva K, Bonafos C, Despax B, Boudou L, Pons P and Teyssedre G 2013 Kelvin force microscopy characterization of charging effect in thin $\mathrm{a}-\mathrm{SiO}_{\mathrm{x}} \mathrm{N}_{\mathrm{y}}: \mathrm{H}$ layers deposited in pulsed plasma enhanced chemical vapor deposition process by tuning the Siliconenvironment J. Appl. Phys. 113204102

[15] Okamoto T, Kitagawa S, Inoue N and Ando A 2011 Electric filed concentration in the vicinity of the interface between anode and degraded $\mathrm{BaTiO}_{3}$-based ceramic capacitor Appl. Phys. Lett. 98072905

[16] Jacquith M, Muller E M and Marohn A 2007 Time-resolved electrostatic force microscopy of charge trapping in polycrystalline pentacene J. Phys. Chem. B 1117711

[17] Ng T N, Silveira W R and Marohn J A 2007 Dependence of charge injection on temperature, electric field, energetic disorder in an organic semiconductor Phys. Rev. Lett. 98 066101

[18] Martin B and Kliem H 2008 Space charge measurements with the scanning Kelvin Probe IEEE Trans. Dielec. Electr. Insul. 15560
[19] Mortreuil F, Villeneuve-Faure C, Boudou L, Makasheva K and Teyssedre G 2017 Charges injection phenomena at metal/ dielectric interface investigated by Kelvin probe force microscopy J. Phys. D: Appl. Phys. 50175302

[20] Faliya K, Kliem H and Dias C J 2016 Investigations of space charge distributions by atomic force microscope IEEE Conf. Proc. of Int. Conf. on Dielectric (ICD) (https://doi.org/ 10.1109/ICD.2016.7547584)

[21] Faliya K, Kliem H and Dias C J 2017 Space charge measurements with Kelvin Probe force microscopy IEEE Trans. Dielec. Electr. Insul. 241913

[22] Zaghloul U, Papaioannou G J, Coccetti F, Pons P and Plana R 2010 A systematic reliability investigation of the dielectric charging process in electrostatically actuated MEMS based on Kelvin probe force microscopy J. Micromech. Microeng. 20064016

[23] Zerweck U, Loppacher C, Otto T, Grafström S and Eng L M 2005 Accuracy and resolution limits of Kelvin probe force microscopy Phys. Rev. B 71125424

[24] Cohen G, Halpern E, Nanayakkara S U, Luther J M, Held C, Bennewitz R, Boag A and Rosenwaks Y 2013

Reconstruction of surface potential from Kelvin probe force microscopy images Nanotechnology 24295702

[25] Mortreuil F, Villeneuve-Faure C, Boudou L, Makasheva K and Teyssedre T 2016 Charges injection investigation at metal/ dielectric interfaces by Kelvin Probe force microscopy IEEE Conf. Proc. of Int. Conf. on Dielectric (ICD) (https://doi. org/10.1109/ICD.2016.7547650)

[26] Villeneuve-Faure C, Boudou L, Makasheva K and Teyssedre G 2016 Atomic force microscopy developments for probing space charge at sub micrometer scale in thin dielectric films IEEE Trans. Dielec. Electr. Insul. 23713

[27] Robertson J 2004 High dielectric constant oxides Eur. Phys. J. Appl. Phys. 28265 